\title{
Totally implantable venous access port systems and associated complications: A single-institution retrospective analysis of 2,996 breast cancer patients
}

\author{
LI MA ${ }^{1}$, YUEPING LIU ${ }^{2}$, JIANXIN WANG ${ }^{1}$, YUAN CHANG ${ }^{1}$, LONG YU ${ }^{1}$ and CUIZHI GENG ${ }^{1}$ \\ ${ }^{1}$ Breast Disease Center; ${ }^{2}$ Department of Pathology, The Fourth Hospital of Hebei Medical University, \\ Shijiazhuang, Hebei 050011, P.R. China
}

Received February 16, 2015; Accepted December 7, 2015

DOI: $10.3892 /$ mco.2016.726

\begin{abstract}
Totally implantable venous access port systems (TIVAPS) are widely used in breast cancer patients. However, complications are frequent and may necessitate device replacement or removal, resulting in additional patient stress and treatment delays. The aim of the present study was to investigate possible risk factors for complications. A total of 2,996 consecutive female breast cancer patients, with a median age of 50.2 years (range, 21.2-85.5 years) were enrolled in this observational, single-centre study between December, 2008 and April, 2014. TIVAPS implantation was principally performed using local anaesthesia and the blind puncture or Seldinger technique through internal jugular or subclavian vein access. A retrospective chart review was conducted to obtain information associated with TIVAPS and patient data. Insertion performed by blind puncture and Seldinger technique had a success ratio of 96.34 and $99.80 \%$, respectively $\left(\chi^{2}=29.905, P<0.001\right)$. However, the success ratio of the puncture technique group was $99.76 \%$ when the TIVAPS was implanted in the right internal jugular vein. The most common complications were late complications, with an overall incidence rate of $5.41 \%(162 / 2,996)$ during the entire device duration. The most common late complications included fibrin formation $(1.84 \%, 55 / 2,996)$, port-related bacteraemia $(1.44 \%, 43 / 2,996)$ and deep vein thrombosis $(0.63 \%, 19 / 2,996)$. No patient died during the study. Our results demonstrated that insertion of TIVAPS by blind puncture or the Seldinger technique through internal jugular or subclavian vein access is convenient, and insertion by the Seldinger technique through the right internal jugular vein is the preferred method. Therefore, TIVAPS is safe for continuous infusional chemotherapy regimens for breast cancer patients.
\end{abstract}

Correspondence to: Professor Cuizhi Geng, Breast Disease Center, The Fourth Hospital of Hebei Medical University, 12 Jiankang Road, Shijiazhuang, Hebei 050011, P.R. China

E-mail: 18531117816@163.com

Key words: observational study, breast cancer patients, totally implantable venous access port systems, complications

\section{Introduction}

Totally implantable venous access port systems (TIVAPS), first introduced in 1982, are currently routinely used in chemotherapy, transfusion, parenteral nutrition and blood sampling for laboratory testing (1-3), as they require no external dressing, allow patient activity and require only monthly flushes of heparinized saline to maintain the patency of the catheter. The use of TIVAPS is required for a number of patients with breast cancer, as the majority of such patients require chemotherapy. In chemotherapy patients, central venous administration is useful for avoiding venous toxicity. Although TIVAPS are generally associated with a lower long-term risk of complications compared with peripherally inserted central catheters and central venous catheters, certain complications associated with their placement and long-term use remain a matter of concern. These complications, including infection, catheter fracture, thrombosis, fibrin formation, port inversion, primary dislocation, rejection reaction, port pocket bleeding and extravasation (4-6), may necessitate device replacement or removal, resulting in additional patient stress and treatment delays.

Several studies on cancer patients have investigated potential risk factors for TIVAPS-related complications, with somewhat conflicting results (3,7-10). The reported experience with TIVAPS in breast cancer patients has been limited to studies including a relatively limited number of patients. To date, no specific guidelines or valid recommendations regarding port complications and associated risk factors have been elaborated. In view of the significance of minimizing complications associated with TIVAPS and the limited data availability on potential risk factors, we initiated a large-scale retrospective study to investigate this issue in the context of routine clinical practice in our breast cancer treatment centre.

\section{Patients and methods}

Patients. The present study included 2,996 adult female patients with breast cancer who underwent insertion of TIVAPS over a 65-month period (December, 2008-April, 2014) at the Breast Disease Center of the Fourth Hospital of Hebei Medical University (Shijiazhuang, China). A retrospective chart review was conducted to obtain information on demographical 
characteristics, surgical and device-related factors, wards acommodating the patients following port system insertion, port system-associated adverse events and biological culture results, when available. We monitored the patients until port removal, death, loss to follow-up, or until April 30, 2014, whichever occurred first. Information regarding patients' age, placement side, vein of insertion and catheter tip location, were also collected.

Insertion and maintenance of port systems. All the systems used consisted of a silastic port with a silicone membrane connected to a silicone rubber catheter (7/8F, Groshong catheter, BARD X-Port isp ${ }^{\mathrm{TM}}$; CR Bard Inc., Murray Hill, NJ, USA). Port insertions were performed in the operating theater by a general surgeon under local anaesthesia, using the blind puncture or the Seldinger technique. For the Seldinger techniques group, first, a uniform B-mode ultrasound device was used (Bard), which wasequipped with a 5-10 MHz linear array transducer to view the internal jugular vein for catheter entry in the neck. Subsequently, a 'micropuncture needle' was used to puncture the vein through an introducer. Following this, a guidewire was inserted into the vein through the needle. The needle was withdrawn over the wire and a 'peel-away sheath' was advanced over the guidewire into the vein. Following removal of the dilator component of the peel-away sheath, the catheter was introduced through the sheath and advanced into position. Finally, the catheter was connected with the port. For the other group, a blind puncture was used to establish venous access near the supraclavicular fossa using a puncture needle and the catheter was subsequently inserted through the needle into position (11). A chest X-ray was routinely performed following catheter placement to visualize the location of its tip and to exclude a pneumothorax. The catheter tip was located at the lower or middle part of the superior vena cava. The TIVAPS were routinely sat in a port pocket fixed on the fascia pectoralis, infraclavicularly, in the deltopectoral groove. Prophylactic antibiotics were not routinely administered. Catheter tip malposition was identified immediately after insertion in 26 port system insertion cases. Adjustments were immediately made to ensure the catheter tip was properly located inside the superior vena cava.

The insertion of the port systems was preceded by skin sterilization with an iodine tincture. The port was flushed with $10 \mathrm{ml}$ of heparinized saline $(100 \mathrm{IU} / \mathrm{ml})$ to detect any possible occlusion or subcutaneous leakage prior to injecting any prescribed agent. The port system was flushed with heparinized saline immediately after each administration and once every 4 weeks thereafter, depending on the follow-up schedule of the patient when the port systems were used less frequently.

Statistical analysis. Data were analyzed using SPSS 13.0 software for Windows (SPSS Inc., Chicago, IL, USA). For the statistical analysis, a two-sided P-value of $<0.05$ was considered to indicate a statistically significant difference. Statistical analysis was performed using the Pearson's Chi-square test or Fisher's exact test in cross tables.

\section{Results}

Population and port characteristics. A total of 2,996 consecutive female breast cancer patients were included in the present
Table I. TIVAPS implantation sites and achievement ratio.

\begin{tabular}{lrl}
\hline Implantation site & $\begin{array}{c}\text { TIVAPS, no. } \\
(\mathrm{n}=2,996)\end{array}$ & $\begin{array}{c}\text { Achievement ratio, } \\
\%(\text { no./total })\end{array}$ \\
\hline Blind puncture & 984 & $96.34(948 / 984)$ \\
Right internal jugular v. & 419 & $99.76(418 / 419)^{\mathrm{a}, \mathrm{b}}$ \\
Left internal jugular v. & 386 & $96.11(371 / 386)^{\mathrm{b}}$ \\
Right subclavian v. & 91 & $95.60(87 / 91)^{\mathrm{b}}$ \\
Left subclavian v. & 88 & $81.81(72 / 88)$ \\
Seldinger technique & 2,012 & $99.80(2,008 / 2,012)$ \\
Right internal jugular v. & 1,199 & $99.92(1,198 / 1,199)$ \\
Left internal jugular v. & 813 & $99.63(810 / 813)$
\end{tabular}

${ }^{\mathrm{a}} \mathrm{P}<0.05$ compared with left internal jugular, right subclavian and left subclavian veins; ${ }^{b} \mathrm{P}<0.05$ compared with left subclavian vein. TIVAPS, totally implantable venous access port systems.

study. The overall follow-up duration was with a mean of 264.0 catheter days [standard deviation $(\mathrm{SD})=112.3$ days; range, 9-1,608 days]. The mean age $\pm \mathrm{SD}$ at inclusion was $48.3 \pm 11.2$ years and the median age was 50.2 years (range, 21.2-85.5 years). As shown in Table I, TIVAPS were implanted in the right internal jugular vein in over half of the patients, followed by the left internal jugular, right subclavian and left subclavian veins in the blind puncture group. Furthermore, $\sim 60 \%$ of the patients were implanted with TIVAPS in the right internal jugular vein in the Seldinger technique group. The blind puncture technique was applied in 984 patients, with a success ratio of $96.34 \%$; the Seldinger technique was used in the remaining patients, with a success ratio of $99.80 \%\left(\chi^{2}=29.905\right.$, $\mathrm{P}<0.001)$. In the blind puncture technique group, the highest success ratio was $99.76 \%$ when TIVAPS was implanted in the right internal jugular vein, and the lowest success ratio was $81.81 \%$ when TIVAPS was implanted in the left subclavian vein (Table I). There was no significant difference between the blind puncture and Seldinger technique groups when TIVAPS was implanted in the right internal jugular vein.

Intraoperative complications. We did not observe any TIVAPS-related deaths in this series. Pneumothorax was observed as a complication of TIVAPS placement in 9 patients $(0.30 \%, 9 / 2,996)$; only 2 patients $(0.07 \%)$ required a tube thoracostomy to treat a large pneumothorax, with no additional morbidity. A total of 2 patients $(0.07 \%, 2 / 2,996)$ experienced hemothorax as a serious complication of the port implant, both of whom were successfully treated with tube thoracostomy and venous anticoagulants; 1 patient experienced an accidental lymphatic fistula during the implant procedure, which did not cause any significant complications; 4 patients experienced port pocket bleeding and were successfully treated with re-implantation; finally, 2 patients required an early revision of the implant for malfunction of the catheter due to primary dislocation.

Early complications. The early complications observed in the present study are listed in Table II. Compared with late complications, early complications were rare $(0.83 \%, 25 / 2,996)$ and the majority occurred within 1 month after the implantation 
Table II. Early complications.

\begin{tabular}{lcc}
\hline Complications & No. $(\%)$ & Actions taken \\
\hline Port pocket bleeding & $7(0.23)$ & $\begin{array}{c}\text { Re-implantation } \\
\text { and local treatment } \\
\text { Re-implantation }\end{array}$ \\
$\begin{array}{l}\text { Primary dislocation } \\
\text { Deep vein thrombosis }\end{array}$ & $\begin{array}{l}1(0.03) \\
4(0.13)\end{array}$ & $\begin{array}{c}\text { Anticoagulation } \\
\text { and port removal }\end{array}$ \\
Pocket infection & $5(0.17)$ & $\begin{array}{c}\text { Antibiotics } \\
\text { and port removal } \\
\text { Thrombolysis }\end{array}$ \\
Fibrin formation & $8(0.27)$ & $\begin{array}{c}\text { and port removal } \\
\text { Total }\end{array}$ \\
\hline
\end{tabular}

Table III. Late complications.

\begin{tabular}{|c|c|c|}
\hline Complication & No. $(\%)$ & Actions taken \\
\hline $\begin{array}{l}\text { Catheter fracture } \\
\text { at the lock }\end{array}$ & $4(0.13)$ & Port removal \\
\hline $\begin{array}{l}\text { Catheter fracture } \\
\text { at the site of puncture }\end{array}$ & $3(0.10)$ & Port removal \\
\hline Pinch-off syndrome & $6(0.20)$ & Port removal \\
\hline Catheter dislocation & $3(0.10)$ & Re-implantation \\
\hline Port-related bacteraemia & $43(1.44)$ & $\begin{array}{l}\text { Antibiotics } \\
\text { and port remova }\end{array}$ \\
\hline Deep vein thrombosis & $19(0.63)$ & $\begin{array}{l}\text { Anticoagulation } \\
\text { and port remova }\end{array}$ \\
\hline Fibrin formation & $55(1.84)$ & $\begin{array}{l}\text { Thrombolysis } \\
\text { and port remova }\end{array}$ \\
\hline Extravasation & $13(0.43)$ & $\begin{array}{l}\text { Port removal anc } \\
\text { local treatment }\end{array}$ \\
\hline Port inversion & $3(0.10)$ & Re-implantation \\
\hline Rejection reaction & $13(0.43)$ & $\begin{array}{c}\text { Re-implantation } \\
\text { or removal }\end{array}$ \\
\hline Total & $162(5.41)$ & \\
\hline
\end{tabular}

of TIVAPS (5 cases occurred within the first 15 days and 18 between 15 days and 1 month, prompting premature device removal). The remaining 2 cases occurred after $>1$ month. The most common early complications were port pocket bleeding and fibrin formation in 7 and 8 cases, respectively. Overall, none of the early complications exceeded $0.3 \%$ in our study.

Late complications. The details of the late complications are presented in Table III. A total of 13 patients suffered catheter fracture, including 4 fractures at the port lock level, 3 at the site of puncture and 6 at the level of the clavicle (pinch-off syndrome). A total of 11 cases presented with a separated catheter tip that had migrated into the heart; in the remaining cases the catheter tip was in the superior vena cava. All the separated catheter tips were removed through the right femoral vein by catheter intervention, with no other significant complications.

Other common late complications included port-related bacteraemia, deep vein thrombosis and fibrin formation. A
Table IV. TIVAPS complications for removal.

\begin{tabular}{lr}
\hline Complications & No. \\
\hline Total & 82 \\
Intraoperative $(\mathrm{n}=5)$ & 2 \\
Hemothorax & 1 \\
Lymphatic fistula & 2 \\
Primary dislocation & \\
Early (n=10) & 5 \\
Pocket infection & 3 \\
Fibrin formation & 2 \\
Deep vein thrombosis & \\
Late (n=67) & 4 \\
Catheter fracture at the lock & 3 \\
Catheter fracture at the site of puncture & 6 \\
Pinch-off syndrome & 15 \\
Port-related bacteraemia & 8 \\
Deep vein thrombosis & 15 \\
Fibrin formation & 13 \\
Extravasation & 3 \\
Rejection reaction &
\end{tabular}

TIVAPS, totally implantable venous access port systems.

total of 43 patients suffered from port-related bacteraemia (1.44\%, 22-81 days of port use). The causative agents were Streptococcus mitis and Staphylococcus epidermidis (40 cases). The majority of the infections were successfully treated with appropriate systemic antibiotics; 15 ports were removed after completing the therapeutic schedule. A total of 90 cases of deep vein thrombosis were detected $(0.63 \%, 35-82$ days of port use) at varying intervals following port placement. Low-molecular weight heparin and venous anticoagulants were administered for varying periods of time. Of the 19 ports, 8 were removed. Finally, 55 cases of fibrin formation occurred (1.84\%, 12-52 days of port use), requiring 25,000 IU urokinase to remove the fibrin from the catheter and restore normal flow through the devices, of which 15 were removed due to malfunction despite treatment. There were also 3 rare late complications in our report, including extravasation, port inversion and rejection reaction, in 13,3 and 13 cases, respectively.

Port removal due to complications. The ports were removed in 82 patients $(2.74 \%, 82 / 2,996)$ due to the complications, with a median port duration of 5.2 months in this group (Table IV). The remaining 2,914 ports remained in situ at the time of the last follow-up, or were removed after completing the therapeutic schedule.

\section{Discussion}

Patients with breast cancer often require continuous infusional chemotherapy and frequent blood sampling. The majority of chemotherapeutic agents are associated with significant venous toxicity and often lead to venous thrombosis or 
thrombophlebitis if peripheral veins are used, which becomes increasingly difficult after multiple courses of chemotherapy and frequent venipunctures. By contrast, central venous catheters in such patients are associated with less venous toxicity and may be used to administer all chemotherapy cycles $(12,13)$. Patients also prefer the cosmetic result of a fully implanted venous device compared with that of a central venous device with external lines. TIVAPS are particularly desirable for patients with active lifestyles and they require less maintenance compared with external tunneled catheters. The majority of studies on TIVAPS have investigated heterogenic populations of patients with various malignancies, which led to conflicting findings (14). To the best of our knowledge, the present study included the most cases who underwent insertion of TIVAPS compared with other related studies, and is the first to focus on breast cancer patients specifically.

In this large monocentric study, TIVAPS appeared to provide long-term safe and reliable intermittent venous access. This conclusion is also in accordance with other previous studies $(1,15,16)$. In our case series, the overall complication rate was $6.86 \%(205 / 1,996)$ over the entire device duration. The average port life was 264 days, despite statistics (some of the patients enrolled in our research were only implanted with the ports for a few days before the study was closed; e.g., the shortest duration was only 9 days) and the vast majority of devices remained in situ and complication-free until the day of follow-up or the end of the treatment. The longest port life was 4.4 years (1,608 days). The complications were divided into 3 main categories: Intraoperative, early (occurring prior to the first chemotherapy application) and late (ocurring after the first chemotherapy application). Intraoperative and early complications were rare, with incidence rates of $0.6 \%$ $(18 / 2,996)$ and $0.83 \%(25 / 2,996)$, respectively. These two categories occur early, as a consequence of catheter insertion. The key reasons for these two types of complications have not been fully elucidated and may include imprudent surgical maneuvers or the body constitution of the patients. The former may be addressed by improving surgical techniques and operating more carefully; however, further studies are required to determine the latter potential factor.

The most common complications were late complications, with an overall incidence rate of $5.41 \%(162 / 2,996)$ during the entire device duration. The most common late complication included fibrin formation $(1.84 \%, 55 / 2,996)$, port-related bacteraemia $(1.44 \%, 43 / 2,996)$ and deep vein thrombosis $(0.63 \%, 19 / 2,996)$. Other malfunctions, including extravasation, rejection reaction, fracture, pinch-off syndrome, dislocation and port inversion, occur very rarely $(1.5 \%, 45 / 2,996)$. Fibrin formation was diagnosed by the physician. As fibrin clot formation is the precursor of thrombosis, it is difficult to precisely discern. In fact, the treatments for fibrin formation and thrombosis are the same in our clinical practice. The diagnosis of port-related bacteraemia was defined as a 10-fold increase in colony-forming units of bacteria/ml in the blood obtained through the device, compared with peripheral blood cultures (17). In addition, if qualitative culture was performed, the diagnosis of bacteremia was established when the positivity of blood culture obtained through the TIVAPS was confirmed prior to the culture obtained from peripheral venous blood (18). Despite the advances in antibiotic therapy and infection control procedures, infections remain the most common complication of TIVAPS in cancer patients (19). The incidence of port-related bacteraemia in previous reports varied considerably, owing to dissimilar patient populations and non-standard definitions. The most commonly detected microorganisms were coagulase-negative staphylococci (CoNS). Microbiological agents that colonize catheter hubs and the skin surrounding the insertion site are the sources of most port-related bacteraemias (20). It has been reported that CoNS adhere to the catheter surface through the production of a microbial biofilm $(21,22)$. CoNS rapidly migrate down the catheters due to capillary action and/or electrostatic attraction between the negatively charged organism and the positively charged subcutaneous tissue (23). Furthermore, any negligence of aseptic procedures during operative and maintenance procedures may be another reason. TIVAP-related bloodstream infections contribute to increased morbidity and mortality (12-25\%), and increase the cost of medical treatment and the duration of hospitalization $(24,25)$. The treatments include removal or retention of the device, antibiotic lock preparations and adaptation according to the identified microorganism. Catheter-related thrombosis was another frequent complication in our study. A prospective study by Lee et al (26) reported catheter-related thrombosis in $4.3 \%$ of cancer patients, which is significantly higher compared with that in our study. There was a report that risk factors for thrombosis included $>1$ insertion attempts (26). However, tumor type, disease stage, platelet count, number of chemotherapeutic cycles or types of chemotherapy were not found to be associated with thrombosis (27). Based on earlier studies, in which a high incidence of catheter-related thrombosis was reported, several studies have been conducted regarding the use of thrombosis prophylaxis in these patient groups. However, no reduction in the incidence of thrombosis was observed in our study. In addition to the abovementioned factors, we also demonstrated that location of the catheter tip and vein of access were highly significantly associated with the incidence of thrombosis, which, to the best of our knowledge, had never been priorly reported. A lower incidence of catheter-related thrombosis was observed when the catheter tip was located in the part of the superior vena cava near the posterior segment of the 8th rib, neighbouring the right atrium. The reason may be associated with eddying of the blood flow due to the valve in the right atrium. Our results also demonstrated that the incidence of thrombosis is higher in patients with subclavian catheters when compared with internal jugular vein catheters. This fact may be explained by the more marked angle the subclavian vein forms with the superior vena cava. Injuries to the endothelium by pushing the catheter downwards during the implantation are more likely if the catheter is inserted through the subclavian vein. The chronic mechanical microtrauma and subsequent local toxicity of the chemotherapeutic agents may lead to further injury of the endothelium and predispose to venous thrombosis. However, the jugular vein, particularly the right jugular vein, follows a relatively straight course to the superior vena cava, which makes it a good candidate for port insertion.

Although this is the largest observational study on the incidence rate and risk factors for TIVAPS focusing on breast cancer patients to date, the study has certain limitations. First, it was a monocentric study and all the cases enrolled 
were breast cancer patients. Second, the incidence of some of the complications, such as extravasation, rejection reaction, fracture, pinch-off syndrome, dislocation and port inversion, was low. As we have little experience and no reliable treatment recommendations, the advantages of TIVAPS must be balanced against the risk of complications and port removal according to each individual case. Third, almost all the TIVAPS were used for chemotherapy in our center. Other applications of TIVAPS were rare. Finally, the majority of the TIVAPS in our study were inserted in the internal jugular vein and only few in the subclavian vein. The bias of the data may affect the observational results.

In summary, the insertion of TIVAPS by blind puncture or the Seldinger technique was found to be safe and convenient, and is favored by both medical oncologists and patients. With the increasing use of continuous infusional chemotherapy regimes and the need for improved quality of life of the patients, a significant increase in the requirement for TIVAPS is likely in the future.

\section{References}

1. Vescia S, Baumgärtner AK, Jacobs VR, Kiechle-Bahat M, Rody A, Loibl S and Harbeck N: Management of venous port systems in oncology: A review of current evidence. Ann Oncol 19: 9-15, 2008.

2. Biffi R, Pozzi S, Agazzi A, Pace U, Floridi A, Cenciarelli S, Peveri V, Cocquio A, Andreoni B and Martinelli G: Use of totally implantable central venous access ports for high-dose chemotherapy and peripheral blood stem cell transplantation: Results of a monocentre series of 376 patients. Ann Oncol 15: 296-300, 2004

3. Chang YF, Lo AC, Tsai CH, Lee PY, Sun S, Chang TH, Chen CC, Chang YS and Chen JR: Higher complication risk of totally implantable venous access port systems in patients with advanced cancer - a single institution retrospective analysis. Palliat Med 27: 185-191, 2013

4. Yildizeli B, Laçin T, Batirel HF and Yüksel M: Complications and management of long-term central venous access catheters and ports. J Vasc Access 5: 174-178, 2004.

5. Biffi R, Orsi F, Pozzi S, Pace U, Bonomo G, Monfardini L, Della Vigna P, Rotmensz N, Radice D, Zampino MG, et al: Best choice of central venous insertion site for the prevention of catheter-related complications in adult patients who need cancer therapy: A randomized trial. Ann Oncol 20: 935-940, 2009.

6. Hartkamp A, van Boxtel AJ, Zonnenberg BA and Witteveen PO: Totally implantable venous access devices: Evaluation of complications and a prospective comparative study of two different port systems. Neth J Med 57: 215-223, 2000.

7. Paleczny J, Banyś-Jafernik B, Gazurek K, Kierpieć K, Szczerba H and Zipser P: Long-term totally implantable venous access port systems - one center experience. Anaesthesiol Intensive Ther 45: 215-222, 2013.

8. Hung M-C, Chen C-J, Wu K-G, Hung GY, Lin YJ and Tang RB: Subcutaneously implanted central venous access device infection in pediatric patients with cancer. J Microbiol Immunol Infect 42: 166-171, 2009.

9. Narducci F, Jean-Laurent M, Boulanger L, El Bédoui S, Mallet Y, Houpeau JL, Hamdani A, Penel N and Fournier C: Totally implantable venous access port systems and risk factors for complications: A one-year prospective study in a cancer centre. Eur J Surg Oncol 37: 913-918, 2011.

10. Hsieh C-C, Weng H-H, Huang W-S, Wang WK, Kao CL, Lu MS and Wang CS: Analysis of risk factors for central venous port failure in cancer patients. World J Gastroenterol 15: 4709-4714, 2009.

11. Li J, Fan YY, Xin MZ, Yan J, Hu W, Huang WH, Lin XL and Qin HY: A randomised, controlled trial comparing the long-term effects of peripherally inserted central catheter placement in chemotherapy patients using B-mode ultrasound with modified Seldinger technique versus blind puncture. Eur J Oncol Nurs 18: 94-103, 2014
12. Lipitz-Snyderman A, Sepkowitz KA, Elkin EB, Pinheiro LC, Sima CS, Son CH, Atoria CL and Bach PB: Long-term central venous catheter use and risk of infection in older adults with cancer. J Clin Oncol 32: 2351-2356, 2014.

13. Linnemann B: Management of complications related to central venous catheters in cancer patients: An update. Semin Thromb Hemost 40: 382-394, 2014.

14. Minassian VA, Sood AK, Lowe P, Sorosky JI, Al-Jurf AS and Buller RE: Longterm central venous access in gynecologic cancer patients. J Am Coll Surg 191: 403-409, 2000.

15. Gallieni M, Pittiruti M and Biffi R: Vascular access in oncology patients. CA Cancer J Clin 58: 323-346, 2008.

16. Dal Molin A, Rasero L, Guerretta L, Perfetti E and Clerico M: The late complications of totally implantable central venous access ports: The results from an Italian multicenter prospective observation study. Eur J Oncol Nurs 15: 377-381, 2011.

17. Biffi R, de Braud F, Orsi F, Pozzi S, Mauri S, Goldhirsch A, Nolè $\mathrm{F}$ and Andreoni B: Totally implantable central venous access ports for long-term chemotherapy. A prospective study analyzing complications and costs of 333 devices with a minimum follow-up of 180 days. Ann Oncol 9: 767-773, 1998.

18. Pittiruti M, Hamilton H, Biffi R, MacFie J and Pertkiewicz M; ESPEN: ESPEN Guidelines on Parenteral Nutrition: Central venous catheters (access, care, diagnosis and therapy of complications). Clin Nutr 28: 365-377, 2009.

19. Beckers MM, Ruven HJ, Seldenrijk CA, Prins MH and Biesma DH: Risk of thrombosis and infections of central venous catheters and totally implanted access ports in patients treated for cancer. Thromb Res 125: 318-321, 2010.

20. Mermel LA: Prevention of intravascular catheter-related infections. Ann Intern Med 132: 391-402, 2000.

21. Groeger JS, Lucas AB, Thaler HT, Friedlander-Klar H, Brown AE, Kiehn TE and Armstrong D: Infectious morbidity associated with long-term use of venous access devices in patients with cancer. Ann Intern Med 119: 1168-1174, 1993.

22. Villain-Guillot P, Gualtieri M, Bastide L and Leonetti JP: In vitro activities of different inhibitors of bacterial transcription against Staphylococcus epidermidis biofilm. Antimicrob Agents Chemother 51: 3117-3121, 2007.

23. Cooper GL, Schiller AL and Hopkins CC: Possible role of capillary action in pathogenesis of experimental catheter-associated dermal tunnel infections. J Clin Microbiol 26: 8-12, 1988.

24. Raad I, Hachem R, Hanna H, Bahna P, Chatzinikolaou I, Fang X, Jiang Y, Chemaly RF and Rolston K: Sources and outcome of bloodstream infections in cancer patients: The role of central venous catheters. Eur J Clin Microbiol Infect Dis 26: 549-556, 2007.

25. Veenstra DL, Saint S and Sullivan SD: Cost-effectiveness of antiseptic-impregnated central venous catheters for the prevention of catheter-related bloodstream infection. JAMA 282: 554-560, 1999.

26. Lee AY, Levine MN, Butler G, Webb C, Costantini L, Gu C and Julian JA: Incidence, risk factors, and outcomes of catheter-related thrombosis in adult patients with cancer. J Clin Oncol 24: 1404-1408, 2006.

27. Tesselaar ME, Ouwerkerk J, Nooy MA, Rosendaal FR and Osanto S: Risk factors for catheter-related thrombosis in cancer patients. Eur J Cancer 40: 2253-2259, 2004. 